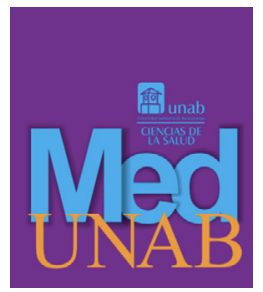

REVISTA DE LA FACULTAD

DE CIENCIAS DE LA SALUD

\title{
Hiperglicinemia no cetósica neonatal, fenotipo atenuado
}

Neonatal nonketotic hyperglycinemia, attenuated phenotype

Hiperglicinemia não-cetótica neonatal, fenótipo atenuado

Alexander Jesús Dellán-Quijada, MD. Esp. ${ }^{1}$ (D), Laura Andrea Useche-Sabogal, MD. Esp. $^{2}$ (D), Oscar Mauricio Espitia-Segura, MD. Esp. ${ }^{3}$ (D) Natalia Penagos-Vargas, MD. Esp. ${ }^{5}$ (D)

1. Médico, Pediatra, Neonatólogo, Especialista en Puericultura. Clínica Santa Teresita del Niño Jesús, Bogotá, Colombia.

2. Médico, Especialista en Pediatría y Neonatología. Clínica Santa Teresita del Niño Jesús, Hospital Militar Central, Bogotá, Colombia

3. Médico, Especialista en Neuropediatría. Fundación HOMI Hospital de la Misericordia, Bogotá, Colombia

4. Médico, Especialista en Neuropediatría. Fundación HOMI Hospital de la Misericordia, Bogotá, Colombia

Correspondencia. Alexander Jesús Dellán Quijada. Clínica Santa Teresita del Niño Jesús. KR 96C \# 21A-43, Bogotá D.C. Email. alexanderdellan@gmail.com

\section{INFORMACIÓN DEL ARTÍCULO:}

Artículo recibido: 19 de mayo de 2020

Artículo aceptado: 17 de junio de 2021

DOI: https://doi.org/10.29375/01237047.3908

Cómo citar: Dellán-Quijada A, Useche-Sabogal LA, Espitia-Segura OM, Penagos-Vargas N. Hiperglicinemia no

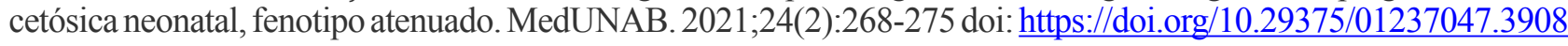

\section{RESUMEN}

Introducción. La Hiperglicinemia no Cetósica (HNC) es un error innato del metabolismo de herencia autosómica recesiva, cuya principal característica es la acumulación de glicina en los fluidos corporales, producido por una falla en el complejo de clivaje enzimático de este aminoácido. Presentación del caso. Presentamos el caso de un recién nacido de 36 semanas, con adaptación neonatal espontánea, sin historia de noxa perinatal ni hipoglicemia documentada, quien tras un corto período de 24 horas presentó deterioro neurológico progresivo, rápida alteración del estado de conciencia hasta el coma y falla ventilatoria. Llamó la atención al ingreso la hipotonía severa generalizada, hiporreflexia, ausencia de reflejos primitivos, con episodios de hipo aislado y movimientos oculares anormales. Ante la sospecha de un error innato del metabolismo se realizó el perfil de aminoácidos donde se evidenció elevación significativa de la glicina, $1417 \mathrm{mmol} / \mathrm{L}$ (referencia 94-553 umol/L). Se solicitaron aminoácidos en líquido cefalorraquídeo, glicina muy elevada $1263 \mathrm{mmol} / \mathrm{L}$ (referencia 3-7 umol/L), con lo que se confirma la sospecha de hiperglicinemia no 
cetósica. Se decidió iniciar manejo con benzoato de sodio y dextrometorfano. La resonancia magnética inicial fue normal, en estudio control se encontraron al igual que en el electroencefalograma hallazgos reportados previamente en la literatura para esta patología. Discusión. La mayoría de los niños con HNC se presentan en el período neonatal o en la primera infancia, y solo los casos más leves se presentan al final de la infancia o la niñez. En las presentaciones de inicio neonatal, el $85 \%$ tiene $\mathrm{HNC}$ grave y el 15\% tiene forma atenuada, como este caso. El diagnóstico de la HNC se hace con base en la sospecha clínica, confirmada por los hallazgos de laboratorio, con la alteración característica de la glicina tanto en plasma como en el LCR y soportada por los hallazgos de las neuroimágenes y electroencefalograma (EEG). Conclusiones. La HNC no es una condición tan inusual, aunque sí posiblemente subdiagnosticada por la forma de presentación tan catastrófica, además porque no produce grandes desarreglos metabólicos de rápido diagnóstico. Por este motivo, ante un paciente con cuadro clínico sugestivo, con coma, alteración respiratoria y convulsiones de difícil manejo, y muy característicamente hipo, debe solicitarse el estudio de aminoácidos en plasma, neuroimágenes y EEG, con el fin de instaurar un manejo temprano.

Palabras claves:

Hiperglicinemia no Cetósica; Glicina; Recién nacido; Epilepsia; Hipo.

\section{ABSTRACT}

Introduction. Nonketotic hyperglycinemia (NKH) is an autosomal recessive innate error of metabolism, whose main characteristic is the accumulation of glycine in body fluids, produced by a failure in the enzymatic cleavage complex of this amino acid. Case Presentation. We present the case of a 36-week-old newborn, with spontaneous neonatal adaptation, no history of perinatal noxa or documented hypoglycemia, who after a short period of 24 hours presented progressive neurological deterioration, rapid alteration of consciousness to coma and ventilatory failure. At admission the patient was noted for severe generalized hypotonia, hyporeflexia, absence of primitive reflexes, with episodes of isolated hiccups and abnormal eye movements. In view of the suspicion of an innate error of metabolism, an amino acid profile was performed, showing a significant elevation of glycine, $1417 \mathrm{umol} / \mathrm{L}$ (reference $94-553 \mathrm{umol} / \mathrm{L}$ ). Amino acids were requested in cerebrospinal fluid, glycine very elevated $1263 \mathrm{umol} / \mathrm{L}$ (reference 3-7 umol/L), confirming the suspicion of nonketotic hyperglycinemia. It was decided to start treatment with sodium benzoate and dextromethorphan. The initial MRI was normal; in the control study, findings previously reported in the literature for this pathology were found, as well as in the electroencephalogram. Discussion. Most children with NKH will display it in the neonatal period or early infancy, with only the mildest cases presenting in late infancy or childhood. In neonatal-onset cases, $85 \%$ have severe $\mathrm{NKH}$ and $15 \%$ have attenuated form, as in this case. The diagnosis of NKH is made based on clinical suspicion, confirmed by laboratory findings, with the characteristic alteration of glycine in both plasma and CSF and supported by neuroimaging and electroencephalogram (EEG) findings. Conclusions. NKH is not such an unusual condition, although it is possibly underdiagnosed because of its catastrophic presentation and because it does not produce major metabolic disorders that are quickly diagnosed. For this reason, in a patient with a suggestive clinical condition, with coma, respiratory alteration and unmanageable seizures, and very characteristically hiccups, the study of amino acids in plasma, neuroimaging and EEG should be requested, in order to establish early treatment.

Keywords:

Hyperglycinemia, Nonketotic; Glycine; Newborn; Epilepsy; Hiccups.

\section{RESUMO}

Introdução. A hiperglicinemia não-cetótica (HNC) éum erro inato do metabolismo de herança autossômica recessiva, cuja principal característica é o acúmulo de glicina nos fluidos corporais, produzido por uma falha no complexo de clivagem enzimática deste aminoácido. Apresentação do caso. Apresentamos o caso de um recém-nascido de 36 semanas, com adaptação neonatal espontânea, sem história de noxa perinatal nem hipoglicemia documentada, que após um curto período de 24 horas apresentou deterioração neurológica progressiva, alteração rápida de consciência até coma e falha ventilatória. Na admissão, eram notáveis a hipotonia grave generalizada, hiporreflexia, ausência de reflexos primitivos, com episódios de soluços isolados e movimentos oculares anormais. Diante da suspeita de erro inato no metabolismo, foi realizado o perfil de aminoácidos, onde foi constatada elevação significativa da glicina, 1417umol/L (referência 94-553 umol/L). Foram solicitados aminoácidos no líquido cefalorraquidiano, glicina muito alta $1263 \mathrm{umol} / \mathrm{L}$ (referência 3-7 umol/L), confirmando a suspeita de hiperglicinemia não-cetótica. Foi decidido iniciar o tratamento com benzoato de sódio e dextrometorfano. A ressonância magnética 
inicial foi normal, tanto em estudo controle quanto no eletroencefalograma, foram encontrados achados previamente relatados na literatura para esta patologia. Discussão. A maioria das crianças com HNC estão no período neonatal ou na primeira infância, e apenas os casos mais leves ocorrem na infância ou na infância tardia. Nas apresentações de início neonatal, $85 \%$ têm $\mathrm{HNC}$ grave e 15\% têm forma atenuada, como neste caso. O diagnóstico de HNC é feito com base na suspeita clínica, confirmada por achados laboratoriais, com alteração característica da glicina tanto no plasma quanto no LCR e apoiado por achados de neuroimagem e eletroencefalograma (EEG). Conclusões. A HNC não é uma condição tão incomum, embora possivelmente seja subdiagnosticada por sua apresentação catastrófica, também por não produzir grandes distúrbios metabólicos que possam ser diagnosticados rapidamente. Por esse motivo, em um paciente com quadro clínico sugestivo, com coma, distúrbios respiratórios e convulsões de difícil manejo, e soluços muito característicos, deve ser solicitado um estudo de aminoácidos no plasma, neuroimagem e EEG a fim de estabelecer um tratamento rápido.

Palavras-chave:

Hiperglicinemia não-Cetótica; Glicina; Recém-nascido; Epilepsia; Soluço.

\section{Introducción}

La Hiperglicinemia no Cetósica (HNC) es un error innato del metabolismo de herencia autosómica recesiva, cuya principal característica es la acumulación de glicina en los fluidos corporales, producido por una falla en el complejo de clivaje enzimático de este aminoácido $(1,2)$.

La glicina es un aminoácido no esencial (1) con acción dual como neurotransmisor excitador e inhibidor. A nivel central, tiene un efecto excitatorio sobre el receptor NMDA en hipocampo y corteza, generando en condiciones patológicas sobreexcitación en estas estructuras y en consecuencia, convulsiones refractarias y lesión cerebral (3). En cordón espinal, el receptor clásico tiene funciones inhibitorias y es el responsable de las apneas, la hipotonía y el hipo en estos pacientes (4).

En general se considera de baja frecuencia, con una incidencia de 1 en 250,000 recién nacidos en Estados Unidos. En otros países donde se ha estudiado activamente, se ha encontrado una incidencia más alta, como en Finlandia $(1: 55,000)$ y en Columbia Británica, Canadá $(1: 63,000)(1,2)$. En Colombia la descripción más grande incluye 20 pacientes con $\mathrm{HNC}$ entre los años 2000 y 2014 en Medellín (5).

La frecuencia encontrada en estudios de alto riesgo ofrece una perspectiva diferente, mostrando que el número de reportes en Colombia es mayor comparado con otras aminoacidopatías que son consideradas comunes en otros países, por ejemplo la fenilcetonuria (1). Considerando esto, en nuestro medio, es importante tener un alto índice de sospecha de la condición frente a los pacientes con compromiso neurológico severo en los primeros días de vida.

A continuación presentamos el caso de una recién nacida con diagnóstico de HNC atenuada de presentación neonatal, su abordaje diagnóstico y terapéutico.

\section{Reporte del caso}

Recién nacido de 36 semanas, con adaptación neonatal espontánea, sin historia de noxa perinatal ni hipoglicemia documentada, quien tras un corto periodo de 24 horas presentó deterioro neurológico progresivo, rápida alteración del estado de conciencia hasta el coma y falla ventilatoria. Al ingreso, el paciente se encuentra en ventilación mecánica, sin asistencia al respirador, escala Glasgow 3 puntos, hipotonía severa generalizada, hiporreflexia, ausencia de reflejos primitivos, con episodios de hipo aislado y movimientos oculares anormales.

Se mantuvo inestable los primeros días, requiriendo soporte inotrópico y ventilación mecánica. Sin embargo, a partir del día 13 de vida, se evidenció recuperación progresiva de la esfera neurológica. Reaparece el reflejo corneal, así como tusígeno y nauseoso, así mismo mejora el tono muscular. A los 15 días de vida se observó mayor reactividad pupilar y reflejos primitivos del recién nacido. De forma progresiva se logró el retiro del soporte ventilatorio e inotrópico y se inició alimentación por succión, con adecuado patrón succión deglución. Persistió con hipo y movimientos oculares anormales. Se asociaron al cuadro posteriormente crisis focales, que se manejaron con fenobarbital y fenitoína con buenos resultados.

En cuanto a la aproximación diagnóstica, se estudió inicialmente con resonancia magnética (RM) cerebral sin evidencia de alteraciones estructurales, marcadores de sepsis que fueron negativos, perfil de electrolitos y glicemia que fueron normales. Solo se documentó en radiografía de tórax cardiomegalia, por lo que se descartó enfermedad de Pompe con Alfa Glucosidasa y CPK que fueron normales. Basados en la clínica y los hallazgos antes mencionados, se sospechó error innato del metabolismo intermediario, se solicitaron gases arteriales, amonio, relación lactato/piruvato, 
aminoácidos en sangre, acilcarnitinas, ácidos orgánicos y cetonas. De estos estudios solo fue anormal el perfil de aminoácidos donde se evidenció elevación significativa de la glicina, 1417umol/L (referencia 94-553). Se solicitaron aminoácidos en líquido cefalorraquídeo, glicina muy elevada $1263 \mathrm{umol} / \mathrm{L}$ (referencia 3-7), con lo que se confirmó la sospecha de hiperglicinemia no cetósica. Se decide iniciar manejo con benzoato de sodio y dextrometorfano.
En este punto se complementaron los estudios con RM cerebral de control que para este punto mostró restricción a la difusión en tractos del tegmento pontomesencefálico, leve restricción a la difusión de cápsula interna, con aumento de señal en T2 e hipoplasia vermiana (Figura 1) y telemetría de cuatro horas, con trazado de fondo de características continuas anormales por ocasionales descargas epileptiformes de ondas agudas centroparietales de predominio izquierdo sin trazado de estallido supresión (Figuras 2 y 3 ).

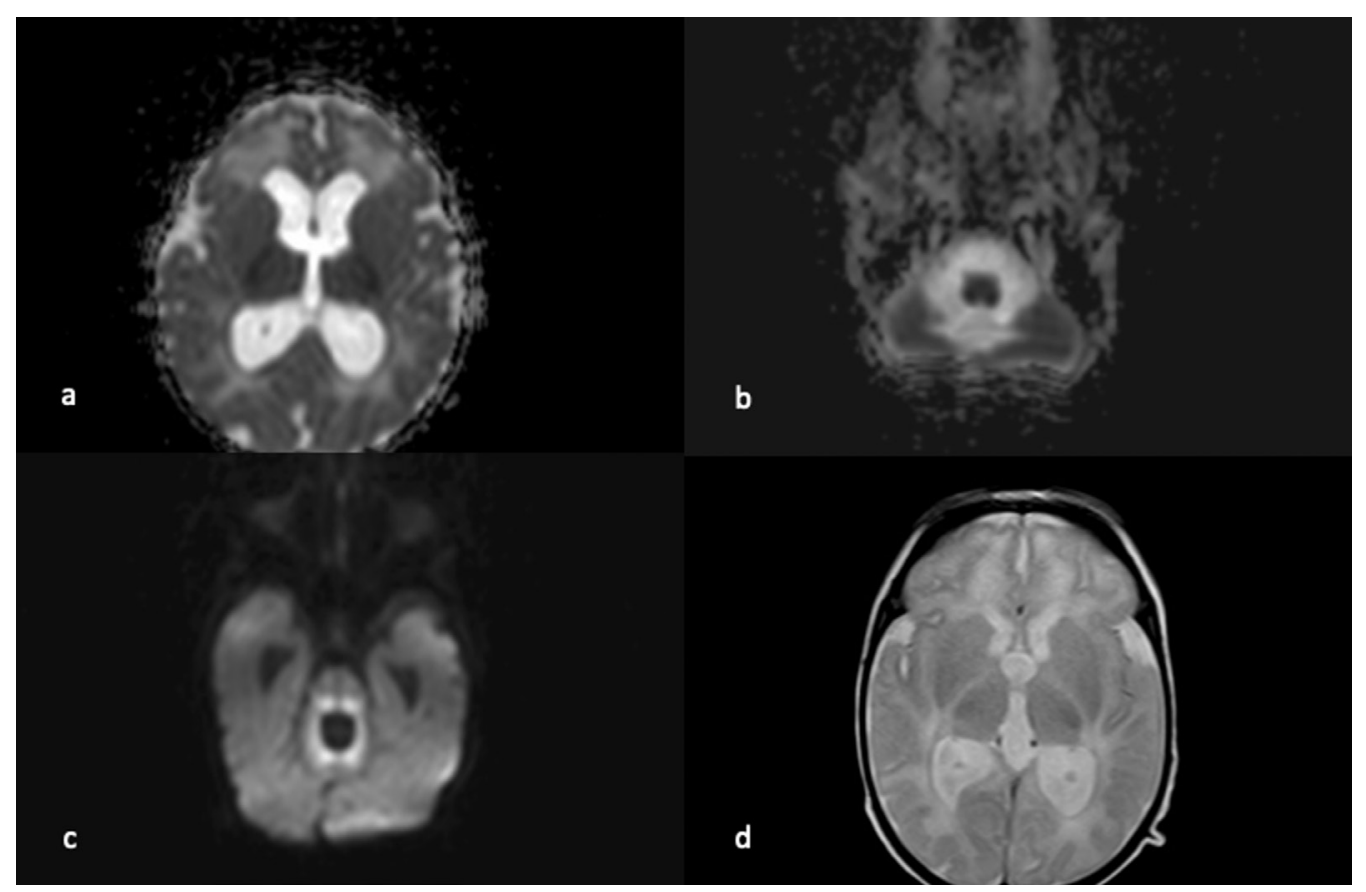

a. Imagen ADC con evidencia de restricción a la difusión de brazo posterior de la cápsula interna bilateral. b y c. Imágenes de difusión (ADC y b1000) con evidencia de restricción a la difusión en tegmento póntico y tractos bulbares dorsales. d. T2 axial: aumento de señal en brazo posterior de la cápsula interna.

Figura 1. Resonancia Magnética Cerebral. Secuencias de difusión ADC, b1000 y T2 axial

Fuente: tomado de la historia clínica del paciente.

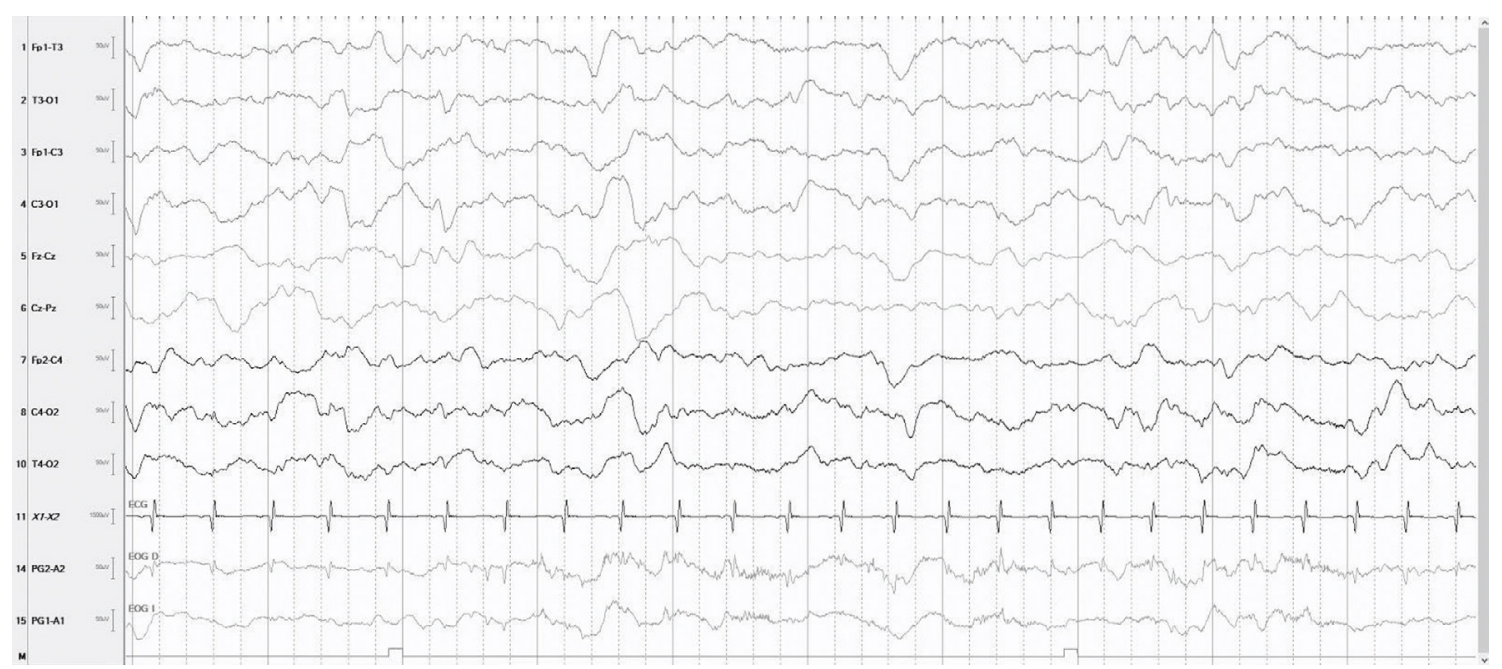

Figura 2. EEG (Sens $7 \mathrm{mcv}, \mathrm{TC} 0.1 \mathrm{~s}, \mathrm{HF} 70 \mathrm{~Hz}$ ) Lentificación intermitente difusa

Fuente: tomado de la historia clínica del paciente. 


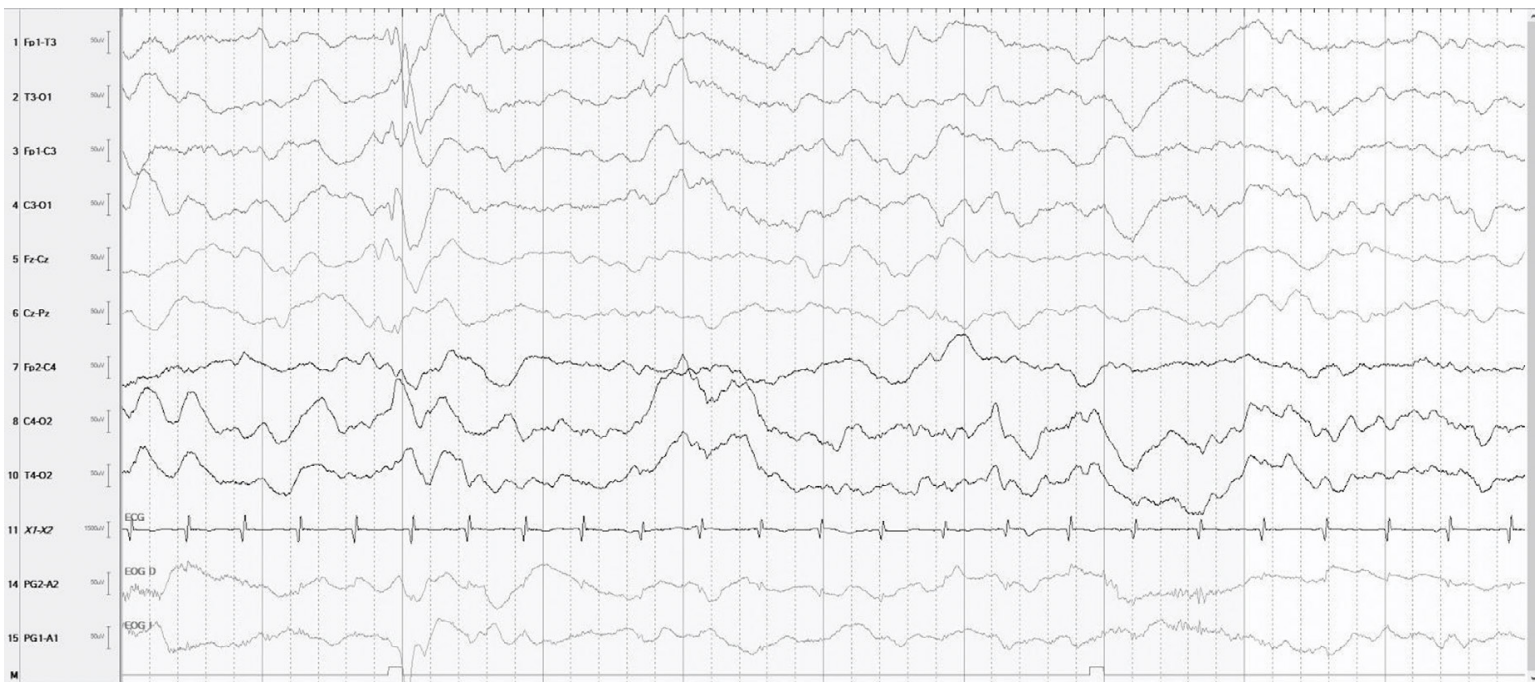

Figura 3. EEG (Sens $7 \mathrm{mcv}$, TC $0.1 \mathrm{~s}$, HF $70 \mathrm{~Hz}$ ) Actividad epiléptica focal centroparietal izquierda

Fuente: tomado de la historia clínica del paciente.

Continúa en seguimiento en otra institución, por epilepsia refractaria, con recurrencia ictal asociada a dificultades en adherencia, particularmente al benzoato de sodio. Durante el seguimiento se estudiaron nuevamente los aminoácidos encontrando una relación de Glicina
LCR/plasma de 0,08 (LCR37/sangre456), así mismo, deterioro en el patrón de EEG, que presenta segmentos de "estallido supresión" (Figura 4) a los 3 meses de edad, que mejoró posteriormente.

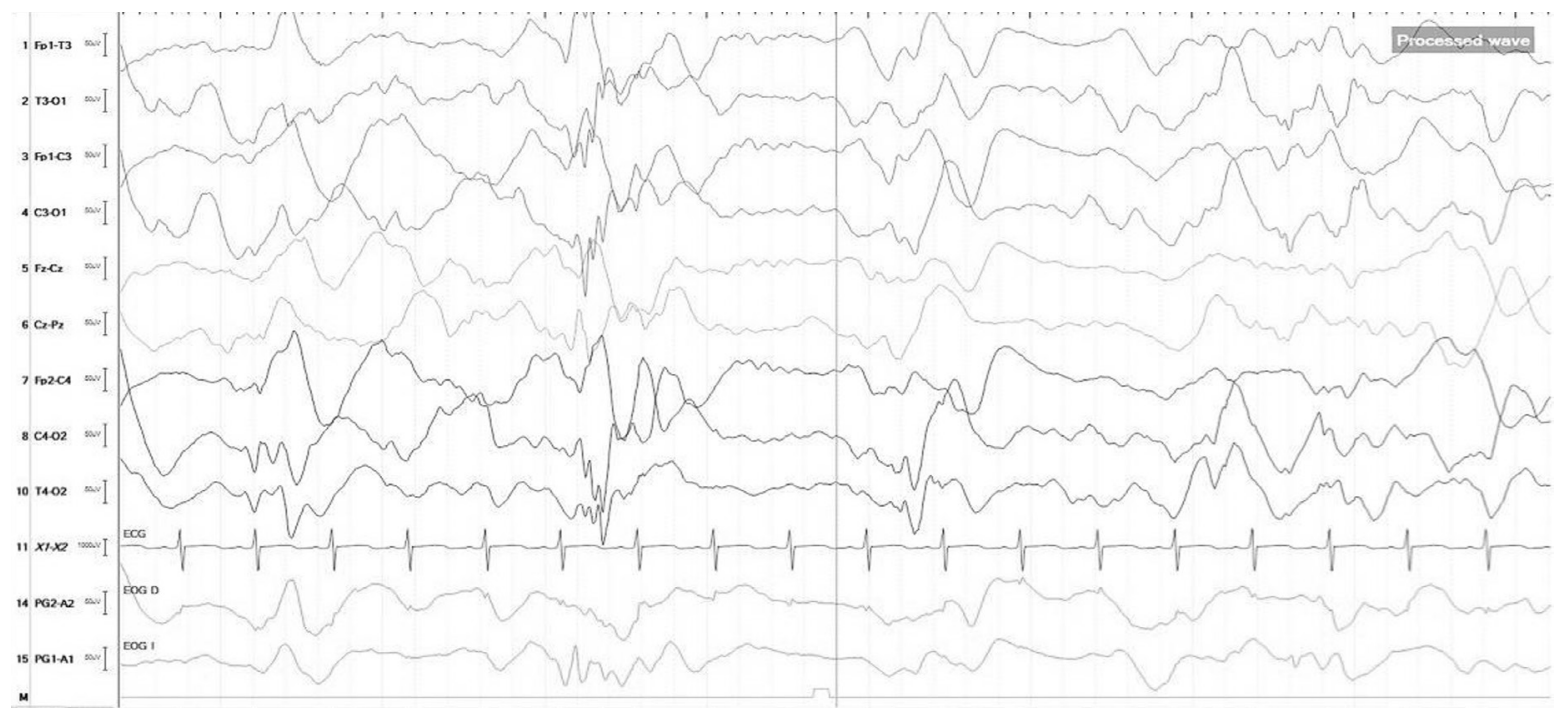

Figura 4. (Sens 7mcv, TC 0.1s, HF $70 \mathrm{~Hz}$ ) Actividad epiléptica y segmentos de estallido-supresión de EEG

Fuente: tomado de la historia clínica del paciente.

\section{Discusión}

La HNC se produce por alteraciones en las enzimas de clivaje de la glicina, las cuales se expresan en hígado, cerebro y placenta, y en niveles bajos en los linfoblastos transformados por el virus Epstein-Barr (1). La actividad enzimática deficiente causa niveles elevados de glicina en plasma y líquido cefalorraquídeo (LCR), con una relación de glicina LCR/plasma elevada (6).
Muchos niños con HNC nacen aparentemente sanos y permanecen asintomáticos por un breve periodo. Luego presentan una progresión rápida de síntomas neurológicos, tales como pobre succión, letargia, convulsiones e hipotonía generalizada. La alteración del control de la mirada vertical se observa con frecuencia como manifestación del efecto inhibidor de la glicina a nivel de tallo. Muchos pacientes se deterioran rápidamente hasta el coma, falleciendo en algunas semanas $(2,7)$. 
La HNC está asociada con un pobre pronóstico con una mortalidad superior al $30 \%$, gran parte en periodo neonatal (8). Los sobrevivientes usualmente tienen retardo psicomotor severo, microcefalia, espasticidad y convulsiones de difícil control (7).

Se describen en la literatura tres formas de presentación de la HNC: 1)neonatal (primeras horas de vida) con letargo que evoluciona a coma profundo e hipotonía. En el $80 \%$ de los bebés, el esfuerzo ventilatorio se reduce en diferentes grados, aunque la mayoría recupera la respiración espontánea al cabo de pocas semanas y algunos muestran una mejoría espontánea en el estado de alerta en el primer mes de vida, a menudo recuperan la succión y la tolerancia a la vía oral (7). El hipo se presenta con frecuencia, así como las convulsiones, aunque su fisiopatología es diferente; el primero, como se mencionó, por el efecto inhibidor de la glicina en tallo (5,7). 2)Infantil (edad $>2$ semanas a 3 meses). Si bien estos bebés no tienen letargo ni coma en los primeros días de vida, a menudo tienen antecedentes de hipotonía desde el principio. Se presentan con retraso en el desarrollo y convulsiones de inicio infantil que pueden ser leves y evolucionan con severidad variable (7). 3)La tardía (edad $>3$ meses) es la forma de presentación más infrecuente. Aunque implica invariablemente retraso en el desarrollo, puede no asociarse con epilepsia (7). La $\mathrm{HNC}$ se clasifica en HNC grave y HNC atenuada según el curso y pronóstico de la enfermedad (6).

En la forma grave los niños no progresan en su desarrollo y tienen una epilepsia refractaria o de difícil manejo. En la forma atenuada, los niños tienen un progreso de desarrollo variable y tienen epilepsia tratable o no tienen epilepsia. Esta forma se divide a su vez en: atenuada severa, en la que los niños tienen un cociente de desarrollo de $<20$ y todos tienen epilepsia; atenuada intermedia, en la que los niños tienen un coeficiente de desarrollo de 20 a 50 y epilepsia fácilmente tratable o no tienen epilepsia; y atenuada benigna, en la que los niños tienen un coeficiente de desarrollo $>50$ y no tienen epilepsia.

La mayoría de los niños con $\mathrm{HNC}$ se presentan en el período neonatal o en la primera infancia, y solo los casos más leves se presentan al final de la infancia o la niñez. En las presentaciones de inicio neonatal, el 85\% tiene $\mathrm{HNC}$ grave y el $15 \%$ tiene forma atenuada, como este caso. En las presentaciones de inicio infantil ( $>2$ semanas - 3 meses), el $50 \%$ tiene $\mathrm{HNC}$ grave y el $50 \%$ tiene $\mathrm{HNC}$ atenuada (6).

El diagnóstico de la HNC se hace con base en la sospecha clínica, confirmada por los hallazgos de laboratorio y soportada por imágenes y electroencefalograma (EEG).
En cuanto a los hallazgos de laboratorio, la combinación de elevación aislada de los niveles de glicina en plasma y LCR (obtenida simultáneamente) por análisis cuantitativo de aminoácidos y una proporción anormal de glicina LCR/plasma hace que la probabilidad de HNC sea alta. La elevación de los niveles de glicina en el LCR en HNC suele ser mayor que la observada en los trastornos que afectan cofactores del sistema de enzimas de escisión de glicina (lipoato, piridoxalfosfato), pero pueden superponerse con los valores en las formas atenuadas. Una relación de glicina LCR/ plasma superior a 0.08 es altamente sugestiva de una forma severa de la condición, mientras en las formas atenuadas suelen verse valores desde 0.04 que podrían dificultar la diferenciación de aquellas condiciones. Debe tenerse presente que la elevación de la glicina en LCR es más importante que la relación, que es solo una medida secundaria y los hallazgos metabólicos hacen, en la práctica, el diagnóstico definitivo en nuestro medio, dada la dificultad en el estudio molecular y enzimático (5). Se espera que el perfil de ácidos orgánicos en la orina sea normal, sin presentar grandes desarreglos en estudio metabólico (2). Pequeñas elevaciones de múltiples ésteres de acilglicina se han reportado ocasionalmente (7).

En las series reportadas, un alto porcentaje $>75 \%$ presenta el patrón de estallido supresión en el EEG, que si bien no es específico, es sugestivo de esta condición (5).En neuroimágenes se reportan la espongiosis generalizada de los tractos ascendentes descendentes de sustancia blanca como los principales rasgos histopatológicos de HNC neonatal (10). En una serie de 24 pacientes de presentación neonatal se encontró restricción a la difusión en todos ellos en brazo posterior de cápsula interna, tallo en su porción anterior, tractos del tegmento posterior y cerebelo, además de hipoplasia del cuerpo calloso (11); y en otros estudios, hipoplasia vermiana y megacisterna magna (2). Estos hallazgos son consistentes en diferentes series y se presentaron en este caso también. En las series reportadas, un alto porcentaje $>75 \%$ presenta el patrón de estallido supresión en el EEG, que si bien no es específico, es sugestivo de esta condición (5).

El pronóstico y el fenotipo de los pacientes con $\mathrm{HNC}$ se ha relacionado tanto con la presentación clínica como con los niveles de glicina en sangre y el LCR, así como la actividad residual del sistema enzimático de clivaje (6). Los niños con HNC severa con frecuencia tienen pocos, avances en el desarrollo y cursan con epilepsia refractaria (7), mientras que, en las formas atenuadas, los niños tienen progresos variables y epilepsia que suele ser más leve o incluso sin esta cuando se trata de un fenotipo bien atenuado (6). El pronóstico, sin embargo, no está relacionado con la edad de inicio, dado que la mayoría de 
los niños con $\mathrm{HNC}$ se presentan en el período neonatal o en la primera infancia, incluso los casos de enfermedad atenuada o leve, por lo cual predecir el pronóstico sobre esta base es inadecuado (6).

Debido a que no hay un tratamiento específico, el manejo actual consiste en reducir los niveles de glicina con benzoato de sodio y bloquear el efecto del exceso de glicina en los receptores N-metil-D-aspartato (NMDA) con dextrometorfano (7). El ácido benzoico se activa a benzoil-CoA, luego se conjuga con glicina para formar hipurato, que se excreta en la orina, por lo tanto, eliminando la glicina y reduciendo los niveles. La dosis de benzoato debe individualizarse con el objetivo de tener niveles de glicina en plasma entre 120 y $300 \mathrm{umol} / \mathrm{L}$. La dosis de benzoato requerida depende de la actividad enzimática residual de escisión de la glicina, y es menor en $\mathrm{HNC}$ atenuada que en la forma grave $(6,12)$. La glicina es un coactivador alostérico del receptor de glutamato tipo NMDA, cuya sobreestimulación se ha planteado como un componente de la patogenia de HNC. El dextrometorfano es un inhibidor débil de este receptor, y su uso puede disminuir las convulsiones en $\operatorname{HNC}(7,12)$. En HNC atenuada, el tratamiento con benzoato de sodio y dextrometorfano mejora el resultado neurocognitivo cuando se instituye tempranamente. Esto contrasta con la falta de eficacia en aquellos pacientes con $\mathrm{HNC}$ grave. Para pacientes en quienes las mutaciones confieren actividad residual, la terapia debe llevarse a cabo temprano y estrictamente para permitir un resultado óptimo (12).

\section{Conclusión}

La HNC no es una condición tan inusual, aunque sí posiblemente subdiagnosticada por la forma de presentación tan catastrófica, además porque no produce grandes desarreglos metabólicos de rápido diagnóstico, como acidosis o hipoglicemia; por el contrario, la alteración bioquímica característica es el aumento en los niveles de glicina, estudio que es especializado y al que puede ser difícil acceder. Por este motivo, ante un paciente con cuadro clínico sugestivo, con coma, alteración respiratoria y convulsiones de difícil manejo, y muy característicamente hipo, debe solicitarse el estudio de aminoácidos en plasma inicialmente, EEG y resonancia de cerebro al menos, con el fin de instaurar tratamientos de forma temprana, teniendo en cuenta que la respuesta es variable y no hay un manejo específico, pero puede ayudar en el control de crisis y en algunos casos, en el neurodesarrollo.

\section{Referencias}

1. Uribe A. Hiperglicinemia no cetósica, un aminoacidopatía frecuente en Colombia. Semilleros med 2016;10(1):103-107. Disponible en: https:// www.researchgate.net/publication/313309765 Hiperglicinemia_No_Cetosica_un_aminoacidopatia frecuente en Colombia

2. Iqbal M, Prasad M, Mordekar S. Nonketotic hyperglycinemia case series. J Pediatr Neurosci [Internet] 2015;10(4):355. https://doi. org/10.4103/1817-1745.174445

3. Poothrikovil RP, Al Thihli K, Al Futaisi A, Al Murshidi F. Nonketotic Hyperglycinemia: Two Case Reports and Review. Neurodiagn J [Internet] 2019 Jul 3;59(3):142-151. https://doi.org/10.1080/21646821.2 $\underline{019.1645549}$

4. Cifuentes Y, Bermudez M, Arteaga C. Encefalopatía neonatal. Algo más que asfixia al nacer. Rev la Fac Med [Internet] 2007;55(2):126-134. Disponible en: https://revistas.unal.edu.co/index.php/revfacmed/ article/view/22059/63881

5. Trujillo J, Tobón S, Ortiz B, Mesa S, Vélez G, Cornejo J. Caracterización clínica, bioquímica e imagenológica en una cohorte de pacientes diagnosticados con hiperglicinemia no cetósica clásica: estudio ambispectivo 2000-2014, Medellín, Colombia. Acta neurol colomb 2014;31(4):378-384. https://doi.org/10.22379/2422402255

6. Swanson MA, Coughlin CR, Scharer GH, Szerlong HJ, Bjoraker KJ, Spector EB, et al. Biochemical and molecular predictors for prognosis in nonketotic hyperglycinemia. Ann Neurol [Internet] 2015 Oct;78(4):606-618. Disponible en: http://doi.wiley. com/10.1002/ana.24485

7. Van Hove JLK, Coughlin C II, Swanson M, Hennermann JB. Nonketotic Hyperglycinemia. 2002 Nov 14 [updated 2019 May 23]. In: Adam MP, Ardinger HH, Pagon RA, Wallace SE, Bean LJH, Mirzaa G, Amemiya A, editors. GeneReviews ${ }^{\circledR}$ [Internet]. Seattle (WA): University of Washington, Seattle; 1993-2021. PMID: 20301531. Disponible en: https://www.ncbi.nlm.nih.gov/books/NBK1357/

8. Hoover-Fong JE, Shah S, Van Hove JLK, Applegarth D, Toone J, Hamosh A. Natural history of nonketotic hyperglycinemia in 65 patients. Neurology [Internet]. 2004 Nov 23;63(10):1847-1853. https://doi. org/10.1212/01.WNL.0000144270.83080.29

9. Applegarth DA, Toone JR. Nonketotic Hyperglycinemia (Glycine Encephalopathy): Laboratory Diagnosis. Mol Genet Metab [Internet]. 2001 Sep;74(1,2):139-146. https://doi.org/10.1006/mgme.2001.3224

10. Butler CJ, Likeman M, Mallick AA. Distinctive Magnetic Resonance Imaging Findings in Neonatal 
Nonketotic Hyperglycinemia. Pediatr Neurol [Internet]. 2017 Jul;72(1):90-91. https://doi. org/10.1016/j.pediatrneurol.2017.03.013

11. Stence NV, Fenton LZ, Levek C, Tong S, Coughlin $\mathrm{CR}$, Hennermann JB, et al. Brain imaging in classic nonketotic hyperglycinemia: Quantitative analysis and relation to phenotype. J Inherit Metab Dis [Internet]. 2019 Mar 20;4(3):438-450. Disponible en: https://onlinelibrary.wiley.com/doi/abs/10.1002/ jimd. 12072
12. Bjoraker KJ, Swanson MA, Coughlin CR, Christodoulou J, Tan ES, Fergeson M, et al. NeurodevelopmentalOutcomeand TreatmentEfficacy of Benzoate and Dextromethorphan in Siblings with Attenuated Nonketotic Hyperglycinemia. J Pediatr [Internet] 2016 Mar;170(1):234-239. https://doi. org/10.1016/j.jpeds.2015.12.027 